\section{The nature of tumour cell proliferation}

SIR - The comments by Erika Coward ${ }^{1}$ on the origins of malignancy were interesting though not entirely novel. Clearly, cancer is not just a matter of abnormal proliferation and it would seem necessary to invoke lesions which do more than alter the levels of regulators otherwise one would merely have hyperplasia. Nevertheless, it is equally evident that the major, and possibly only, defect which is common to all transformed cells is that they replicate when they should not do so. Such an effect can be induced in a variety of ways, each of which could produce a unique functional change, while operating through the same mechanism². The ability to totally inhibit proliferation would be a major advance but it might not overcome the effects of the altered properties, for example, increased hormone production.

Bell $^{3}$, has considered some aspects of altered proliferation consequent upon changes in the levels of regulators, both inhibitory and nitrogenic. On the other hand, as a result of my deliberations on the nature of proliferation processes 2,4 , I have suggested ${ }^{5,6}$ that the aberrant behaviour of cancer cells in most cases is due to an altered threshold with respect to regulator levels - decreased with respect to mitogens, raised for inhibitors. (A threshold is required by the cell cycle concept I have developed ${ }^{2.5}$, although it may vary in magnitude and can be zero in particular instances. Its existence provides an explanation for cell density inhibition of proliferation while an altered threshold accounts for the often observed effect thereon of the malignant transformation, namely the ability of such cells to attain much higher densities ${ }^{5}$.) At the same time, I also briefly considered the effects of altered levels of regulators on surrounding normal tissue cells ${ }^{5}$.

There is no evidence that tumour cells in general proliferate faster than the corresponding normal cells. However, although taking different approaches, both Bell and I concluded that a critical tumour size might exist above which the growth may increase more rapidly than hitherto (one possible explanation for latency). My studies ${ }^{5}$ also indicated the possibility that tumours of limited growth potential might arise as a result of lesions which increase the uptake ${ }^{7}$ or binding of regulators, whereas those affecting thresholds could (depending on the magnitude of the change) yield tumours more malignant with respect to growth potential.

A question of major importance to Coward's considerations, however, is whether or not regulation in vivo is ever effected by a single agent. Culture studies clearly indicate that the replicative ability of cells can be affected by a wide range of normal agents, including various hormones. Recent studies have emphasized that transformation is often associated with positive growth factors (mitogens) rather than inhibitors. I have proposed a mechanism for the interaction of regulators ${ }^{2}$ and, on that basis, suggested $^{6}$ (in contrast to the chalone approach) that attempts be made to control the proliferation of tumour cells by the use of a combination of such agents rather than just one (thereby limiting side effects on the replication of normal cells). However, the available data fail to indicate which regulators should be used, and at what concentrations, in any particular instance. It would thus still seem necessary to establish suitable regimes by trial and error, as is the usual procedure.

Another problem is that of heterogeneity in the cells of a tumour. Such heterogeneity implies that the response to growth regulators will also be heterogeneous ${ }^{5}$. As a consequence, treatment by the methods proposed by Coward and by myself, are likely to meet the same limitations in this direction as present approaches. On the other hand, they may be less toxic for the patient and might not produce the opposing adaptive responses.
SIR - Hayashi and Munakata ${ }^{1}$ suggest that the four bases in DNA might be represented by four musical notes. We prefer a graphic representation, where the four bases are displayed in a line extension format: values attributed to the four bases G,A, $T$ and $C$ being $+2,+1,-1$ and -2 . In the example (below), 100 nucleotides of coding sequence from a recent paper ${ }^{2}$ have been converted into this format: significant features of the sequence ("TATA" transcription signal - in fact TATTAT here; start site of transcription; translation initiation codon ATG) are indicated.

Such a presentation has several interesting features. First, particular sequence patterns are readily discernible: purine $(A, G)$ rich stretches appear exclusively above the line and pyrimidine $(C, T)$ stretches below. $G / C$ and $A / T$ rich regions have extended and retracted aspects respectively. Second, restriction sites appear (usually) as simple

Interestingly, accompanying Coward's letter was another by Schleifer et al. ${ }^{8}$ on the effects of psychological factors on the immune response. That brings to mind the old issue of whether or not the mental state can affect tumour formation and development, a topic about which there has been much (often subjective) discussion. There is much data showing that various hormones can regulate cell proliferation, both negatively and positively. It thus seems reasonable to accept that any process modulating hormonal levels, such as stress, is potentially able to modify tumour growth rate (if not transformation), although the actual dependencies are likely to be complex and individualistic.

D. A. GILBERT

University of the Witwatersrand, Johannesburgh, 2001,

South Africa

1. Coward, E. Nature 310, 455 (1984).

2. Gilbert, D.A. Biosystems 10, $227-233$ (1978).

3. Bell, G.I. Science 192, 369-372 (1976).

4. Gilbert, D.A. S. Afr. J. Sci. 77, 541-546 (1981)

5. Gilbert, D.A. Biosystems 9, 215-228 (1977).

6. Gilbert, D.A.S. Afr. J. Sci. 78, $48-49$ (1978)

7. Holley, R.W. Nature 258, 487-490 (1975).

8. Schleifer, S.J., Keller, S.E. \& Stein, M. Nature 310, 456 (1984).

\title{
Machine-readable DNA sequences
}

rotationally-symmetrical patterns which are rapidly picked out by the eye. Third, it takes little time to determine, for instance, that the dinucleotide pair $C$ followed by $G$ is, as expected, depleted. Fourth, to convert to the complementary strand the diagram need only be rotated through the horizontal axis.

Such a line extension format is inherently machine-readable, and may permit suitably equipped readers to transfer sequences directly from the printed page to an information retrieval system.

\section{AFRC Animal Breeding Research}

R. LATHE

R. FINDLAY

Organisation,

West Mains Road,

Edinburgh, UK

1. Hayashi, K. \& Munakata, N. Nature 310, 96 (1984) 2. Gillies, S.D. et al. Nature 310, 594 (1984)

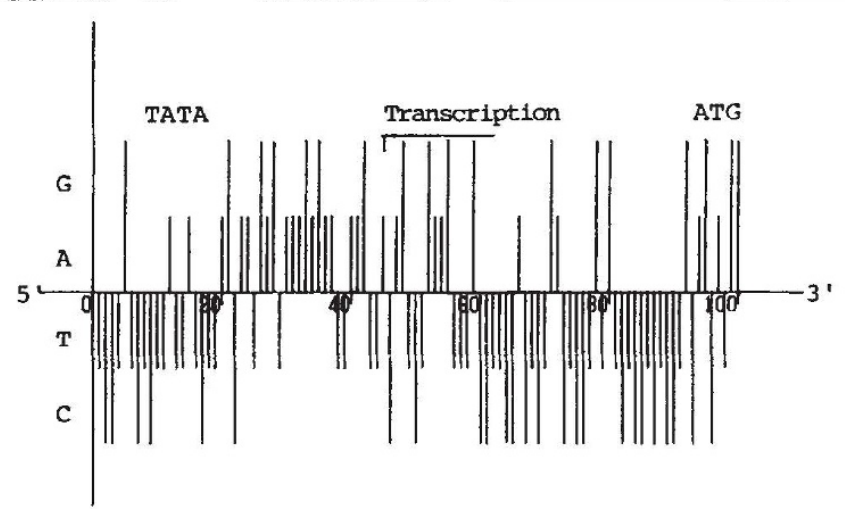

A sequence of 100 nucleotides from a mouse major histocompatibility complex gene, $E_{\beta}$ 